\title{
THE PROPERTIES AND STABILITY OF SELF-GRAVITATING, POLYTROPIC SPHERES WITH $\gamma=1$ TO 1.4 SPECIFIC HEAT RATIOS
}

\author{
A. C. Raga ${ }^{1}$, J. A. Osorio-Caballero ${ }^{1}$, R. S. Chan ${ }^{1}$, A. Esquivel $^{1}$, A. Rodríguez-González ${ }^{1}$, V. Lora $^{2}$, \\ and J. C. Rodríguez Ramírez ${ }^{3}$
}

Received November 19 2019; accepted December 62019

\begin{abstract}
We study self-gravitating, hydrostatic spheres with a polytropic equation of state $P \propto \rho^{\gamma}$ (where $\gamma$ is the specific heat ratio of the gas), considering structures with $\gamma \approx 1$ as a model for molecular cloud cores with small departures from isothermality. We derive the properties (i.e., mass, radius and center to edge density ratio) as a function of $\gamma$ for the maximal stable sphere through an application of "Bonnor's stability criterion". We find that in the $\gamma=1 \rightarrow 4 / 3$ range the mass of the maximal sphere (for a given central temperature) is almost constant, and that its radius and center to edge density ratio are growing functions of $\gamma$. We therefore have maximal stable, self-gravitating spheres with similar masses, but with increasing center to edge density contrasts for increasing departures from isothermality.
\end{abstract}

\section{RESUMEN}

Estudiamos esferas auto-gravitantes hidrostáticas con una ecuación de estado politrópica $P \propto \rho^{\gamma}$ (donde $\gamma$ es el cociente de calores específicos del gas), considerando estructuras con $\gamma \approx 1$ como modelos de núcleos moleculares con pequeñas desviaciones del caso isotérmico. Derivamos las propiedades (masa, radio y cociente de densidades centro a borde) como función de $\gamma$ para la esfera estable máxima, a través de una aplicación del "criterio de estabilidad de Bonnor". Encontramos que en el intervalo $\gamma=1 \rightarrow 4 / 3$ la masa the la esfera máxima (para una temperatura central dada) es casi constante, y que su radio y cociente de densidades centro a borde son funciones crecientes de $\gamma$. Por esto, tenemos esferas autogravitantes máximas con masas similares pero con contrastes de densidad centro a borde crecientes para mayores desviaciones respecto del caso isotérmico.

Key Words: ISM: clouds - ISM: kinematics and dynamics — stars: formation

\section{INTRODUCTION}

It is widely thought that the gravitational collapse of dense molecular cloud cores results in the formation of stars (see, e.g., Shu 1977). Detailed calculations of the thermal structure of molecular cores show that over a large radial range they have only small temperature variations (see, e.g., Falgarone \& Puget 1985), so that their hydrostatic structure can be modeled as an isothermal, self-gravitating, sphere.

\footnotetext{
${ }^{1}$ Instituto de Ciencias Nucleares, UNAM, Ciudad de México, México.

${ }^{2}$ Instituto de Radioastronomía y Astrofísica Teórica, UNAM, Morelia, Michoacán, México.

${ }^{3}$ Instituto de Astronomia, Geofísica e Ciências Atmosféricas, Universidade de São Paulo, SP, Brasil.
}

In this paper, we study self-gravitating spheres with a polytropic equation of state $\left(P \propto \rho^{\gamma}\right.$, where $P$ is the pressure, $\rho$ the density and $\gamma$ the specific heat ratio of the gas) with $\gamma$ values close to 1 , meant as models for cloud cores with small departures from isothermality. We first compute the density profiles of these hydrostatic structures, and then evaluate their stability to radial perturbations.

We use the method of Bonnor (1956) for determining the stability of the isothermal, selfgravitating sphere. Bonnor (1956) considers a sphere that has a fixed mass $M$ and an outer radius $R_{e}$ at which the self-gravitating structure is in contact with a much hotter environment with a pressure $P_{e}$. He then calculates the variation in the outer pressure (or density) of this fixed mass sphere as a function of a 
changing outer radius $R_{e}$ (assuming that the inner structure adjusts instantaneously to the steady, selfgravitating configuration). If the outer density $\rho_{e}$ (or, alternatively the pressure $P_{e}$, which is a monotonically increasing function of $\rho_{e}$ ) satisfies the condition

$$
\frac{d \rho_{e}}{d R_{e}}<0,
$$

the sphere will be stable. In the case of the isothermal sphere, the sphere is stable for small radii, and becomes unstable (i.e., $d \rho_{e} / d R_{e}$ becomes positive) at a radius $R_{S}$. Isothermal spheres with $R_{e}>R_{S}$ are therefore unstable to radial perturbations.

Bonnor (1958) extended his analysis to the case of self-gravitating spheres of arbitrary polytropic index $n=1 /(\gamma-1)$. He derived (from the numerical density stratifications of Emden, 1907) the values of the maximum stable radius $R_{e}$ for the cases with $n=4,4.5,4.9,5$ and 6 . Bonnor's criterion has also been applied to hydrostatic structures with thermal properties calculated using an energy equation with appropriate heat source and sink terms (see Falgarone \& Puget 1985).

Bonnor (1958) finds that self-gravitating, polytropic spheres with $\gamma>4 / 3$ are inconditionally stable to radial perturbations. Because of this, in the present paper we limit ourselves to polytropes with $1 \leq \gamma<4 / 3$. The stability of polytropes with different $\gamma$ values has been described more recently by Horedt (2013).

An interesting problem is whether or not Bonnor's stability criterion gives "correct" results, where by "correct" we mean reproducible by timedependent, hydrodynamic collapse simulations. Until quite recently, hydrodynamic simulations of the collapse of isothermal spheres did not reproduce (at least, not in a quantitative way) the results found from Bonnor's criterion. Hunter (2001) speculated that this lack of agreement was probably the result of a lack of accuracy in the hydrodynamic simulations. More recently, Raga et al. (2013) computed more precise simulations which reproduced the stability found from Bonnor's (1956) criterion for the collapse of the isothermal sphere. Even though it has not yet been proven, in this paper we will assume that Bonnor's (1958) stability criterion for arbitrary $\gamma$ also predicts successfully the stability limits found from time-dependent hydrodynamical collapse calculations.

The paper is organized as follows. In $\S 2$ we present the Lane-Emden equation and give a summary of the analytic properties of its solutions for specific heat ratios in the $\gamma=1 \rightarrow 4 / 3$ range. In
$\S 3$ we describe the density and mass distributions obtained from numerical integrations of the LaneEmden equation. In $\S 4$, we apply Bonnor's (1958) stability criterion to these solutions, and derive the properties of the maximal stable sphere as a function of $\gamma$. Finally the results are summarized in $\S 5$.

\section{THE LANE-EMDEN EQUATION}

\subsection{General Considerations}

The hydrostatic equation for a self-gravitating, nonmagnetized spherical cloud is:

$$
\frac{d}{d R}\left(\frac{R^{2}}{\rho} \frac{d P}{d R}\right)=-4 \pi G \rho,
$$

where $R$ is the spherical radius, $P$ the gas pressure, $\rho$ the density and $G$ the gravitational constant.

We now assume that we have a polytropic law

$$
P=C \rho^{\gamma},
$$

with constant $C$ and $\gamma$. Then, equation (2) takes the form

$$
\frac{d}{d R}\left(R^{2} \rho^{\gamma-2} \frac{d \rho}{d R}\right)=-\frac{4 \pi G}{\gamma C} R^{2} \rho
$$

which receives the name of "Lane-Emden equation".

As we are thinking of modelling a gas cloud, possible values of $\gamma$ are $5 / 3$ (for an atomic/ionic, non-radiative gas), $7 / 5$ (for diatomic molecules) and 1 (for an isothermal situation). Another interesting value is $4 / 3$, corresponding to a gas of ultrarelativistic fermions (relevant for degenerate stellar cores).

In a stratified cloud, there is in principle no reason for $C$ in equation (3) to be independent of radius (individual fluid parcels will evolve satisfying equation 3 , but do not necessarily share the same value of $C$ ). A unique value of $C$ is obtained for a "well mixed" gas cloud, in which mixing due to slow turbulent motions produces a stationary stratification with a constant specific entropy. The existence of such a mixing is required for modelling gas clouds with the polytropic Lane-Emden equation (equation 4).

Finally, we should note that many times the solutions to Lane-Emden's equations are identified with the "polytropic index" $n=1 /(\gamma-1)$ (which appears naturally under some traditional changes of variables used for this equation). However, we will use $\gamma$ (and not $n$ ) throughout the present paper. 


\subsection{The "Small R", Non-Singular Solution}

We assume that we have a non-singular, "flattopped" solution, with $(d \rho / d R)_{0}=0$ in the cloud center. Proposing a second-order Taylor series for the $\rho$ as a function of $R$, inserting it in equation (4) and equating terms with the same powers of $R$ one straightforwardly obtains:

$$
\frac{\rho}{\rho_{0}}=1-\left(\frac{R}{R_{c}}\right)^{2},
$$

where $\rho_{0}$ is the central density and

$$
R_{c} \equiv \sqrt{\frac{3 \gamma C \rho_{0}^{\gamma-2}}{2 \pi G}}
$$

is the "core radius" of the density stratification.

For the variables $r=R / R_{c}$ and $\rho^{\prime}=\rho / \rho_{0}$ the Lane-Emden equation takes the dimensionless form:

$$
\frac{d}{d r}\left[r^{2}\left(\rho^{\prime}\right)^{\gamma-2} \frac{d \rho^{\prime}}{d r}\right]=-6 r^{2} \rho^{\prime},
$$

with second-order solution $\rho^{\prime}=1-r^{2}$.

It is possible to construct higher order solutions by proposing appropriate Taylor expansions for the density as a function of radius. However, it is a well known problem that for $\gamma \approx 1$ the resulting series solutions converge to the exact solution only for $R<R_{c}$ (see, e.g., Chandrasekhar 1967).

We should note that the core radius $R_{c}$ used for the adimensionalization (see equations 6 and 7 ) has a clear dependence on $\gamma$. In order to compare solutions with different $\gamma$ values, it is possible to choose solutions with the same central isothermal sound speed:

$$
c_{0}=\sqrt{\frac{P_{0}}{\rho_{0}}}=\sqrt{C \rho_{0}^{\gamma-1}},
$$

where the first equality is the definition of the isothermal sound speed and the second equality is obtained using equation (3). Substituting into equation (6) we then obtain

$$
R_{c}=\sqrt{\frac{3 \gamma c_{0}^{2}}{2 \pi G \rho_{0}}}=\gamma^{1 / 2} R_{c, 1},
$$

where $R_{c, 1}$ is the core radius of the isothermal $(\gamma=1)$ solution with the same central sound speed $c_{0}$.

Therefore, for comparing solutions with different $\gamma$ values but with the same central temperature (corresponding to a sound speed $c_{0}$ ), we plot the solutions as a function of

$$
\frac{R}{R_{c, 1}}=\gamma^{1 / 2} \frac{R}{R_{c}}=\gamma^{1 / 2} r,
$$

see equations (7) and (9).

The mass of the self-gravitating spheres as a function of the spherical radius is given by:

$$
M(R)=4 \pi \int_{0}^{R} R^{\prime 2} \rho\left(R^{\prime}\right) d R^{\prime}=4 \pi \rho_{0} R_{c}^{3} m\left(R / R_{c}\right),
$$

where

$$
m(r)=\int_{0}^{r} r^{\prime 2} \rho^{\prime}\left(r^{\prime}\right) d r^{\prime}
$$

is the dimensionless mass. It is straightforward to see that $\gamma^{3 / 2} m(r)$ is the mass normalized to $4 \pi \rho_{0} R_{c, 1}^{2}$ (where $R_{c, 1}$ is the core radius of the $\gamma=1$ solution, see above).

\subsection{The Singular Solutions}

In order to obtain the singular solutions, one inserts a proposed solution $\rho_{\text {sing }}=A / R^{p}$ into the LaneEmden equation (4). Equating the resulting powers of $R$ and multiplying constants one obtains:

$$
\begin{gathered}
p=\frac{2}{2-\gamma} \\
A=\left[\frac{\gamma C}{2 \pi G} \frac{4-3 \gamma}{2(2-\gamma)^{2}}\right]^{1 /(2-\gamma)} .
\end{gathered}
$$

Introducing a similar power law into the dimensionless Lane-Emden equation (7) one obtains:

$$
\rho_{\text {sing }}^{\prime}=\left[\frac{4-3 \gamma}{2(2-\gamma)^{2}}\right]^{1 /(2-\gamma)} r^{-2 /(2-\gamma)} .
$$

Clearly, these singular solutions exist only for $\gamma<4 / 3$.

From numerical solutions of the Lane-Emden equation, it is found that for $\gamma<1.2$ the non-singular solution (with the small $r$ solution $\rho^{\prime}=1-r^{2}$ and large $r$ regime obtained by numerically integrating equation 7) converges to the singular solution for $r \gg 1$. For $\gamma=6 / 5=1.2$ (corresponding to a polytropic index $n=5$ ), the Lane-Emden equation has the analytic, non-singular solution

$$
\rho^{\prime}=\left(1+\frac{r^{2}}{3}\right)^{-5 / 2}
$$

This solution has a large $r$ behaviour of the form $\rho^{\prime} \propto r^{-5}$, clearly not matching the singular solution (which for $\gamma=6 / 5$ has a shallower $\rho^{\prime} \propto r^{-5 / 2}$ dependence, see equation 15). The transition between 
the large $r$, singular solution convergence obtained for $\gamma<6 / 5$ and the $\gamma=6 / 5$ solution (given by equation 16) is explored in $\S 3$.

For $\gamma>1.2$, the numerical non-singular solution of the Lane-Emden equation has a density that goes to zero at a finite radius. Therefore, the non-singular solution has a $r \gg 1$ convergence to the singular solution only for $\gamma<6 / 5$. The nature of this convergence is described in the following subsection.

\subsection{The $\gamma\langle 6 / 5, r \gg 1$ Convergence to the Singular Solution}

It is customary to write

$$
\rho^{\prime}(r)=\rho_{\text {sing }}^{\prime}(r)[1+q(r)],
$$

and use equation (7) to obtain a differential equation for the fractional deviation $q(r)$ from the singular solution. Assuming that $q(r), \dot{q}=d q / d r$ and $\ddot{q}=d^{2} q / d r^{2} \ll 1$, this differential equation can be linearized to obtain:

$$
\frac{2(4-3 \gamma)}{2-\gamma}(q+R \dot{q})+R^{2} \ddot{q}=0,
$$

which for $a>3-2 \sqrt{2}$ has the general solution

$$
q(r)=\frac{B}{r^{a}} \cos (\Gamma \ln r+\phi),
$$

where $B$ and $\phi$ are arbitrary constants,

$$
a=2 \frac{4-3 \gamma}{2-\gamma}
$$

and

$$
\Gamma=\frac{1}{2} \sqrt{6 a-a^{2}-1} .
$$

It is straightforward to see that the $a>3-2 \sqrt{2}$ condition (necessary for equation 19 to be valid, see above) is satisfied for the $\gamma=1 \rightarrow 1.2$ range.

Therefore (for all values of $\gamma$ in the $1 \rightarrow 1.2$ range) the large $r$ convergence to the singular solution is oscillatory (going as $\cos \Gamma \ln r$ ). This oscillatory behaviour has an amplitude that decreases as $r^{-a}$ (see equations 19-21).

The linear problem which we have presented here does not allow us to fix the values of the $B$ and $\phi$ constants (see equation 19). These constants have to be determined by fitting equation (17), calculated with the linear $q$ solution (see equation 19), to the nonsingular solution calculated with a numerical integration of the Lane-Emden equation (see equation 7). This fitting can be done to a quite high level of accuracy (see, e. g., the work of Ito et al. 2018 for the $\gamma=1$ solution).

\section{NUMERICAL INTEGRATIONS}

We have numerically integrated the dimensionless Lane-Emden equation (equation 7) starting from the quadratic solution $\rho^{\prime}=1-r^{2}$ at small $r$. The results obtained for several values of $\gamma$ in the $1 \rightarrow 1.2$ range are shown in log-log plots in Figure 1.

For $r<1$, all of the logarithmic density distributions are quite flat, with $\rho^{\prime} \approx 1$. For $r>1$, the $\gamma=1$ and 1.5 distributions start a steeper decrease, and approach the corresponding singular solutions (shown with the straight, dashed lines) with the oscillatory convergence described in $\S 2.3$. These oscillations have larger amplitudes for solutions of increasing $\gamma$.

The bottom panel of Figure 1 shows solutions with $\gamma \approx 1.2$ values. The singular $\gamma=1.2$ solution (not distinguishable from the $\gamma=1.190$ and 1.199 singular solutions at the resolution of the plot) is shown with a dashed line, and the non-singular $\gamma=1.2$ solution (obtained either from the numerical integration or from equation 16) is shown with the red line. It is clear that the $\gamma=1.190$ and $\gamma=1.199$ solutions follow the (analytic) $\gamma=1.2$ solution out to $r_{t} \approx 10$ and 100 (respectively), and then have an oscillatory transition to the singular solution. We find that the transition radius $r_{t}$ becomes progressively larger as $\gamma \rightarrow 1.2$. Solutions with $\gamma \approx 1.2$ have been previously studied by Horedt (2013).

Figure 2 shows logarithmic depictions of the $\rho^{\prime}(r)$ solutions obtained for $\gamma$ values in the $6 / 5 \rightarrow 4 / 3$ range. For all of the $\gamma>1.2$ solutions we obtain densities that go to zero at a finite dimensionless radius $r_{\max }=R_{\max } / R_{c}$ (which can be straightforwardly determined from the numerical integrations).

Finally, in Figure 3 we show the inner regions of density $\rho / \rho_{0}$ and mass $\gamma^{3 / 2} m$ (see equation 12 ) stratifications with $\gamma$ in the $1 \rightarrow 4 / 3$ range, as a function of the radius $\gamma^{1 / 2} r=R / R_{c, 1}$, normalized to the core radius of the $\gamma=1$ solution (see equations 9-10). The results shown illustrate the differences between self-gravitating spheres with different $\gamma$, but with the same central density $\rho_{0}$ and isothermal sound speed $c_{0}$. We see that the lower $\gamma$ solutions have lower densities and masses within the core radius, and higher densities and masses at large radii.

\section{THE RADIAL STABILITY OF THE SELF-GRAVITATING SPHERES}

\subsection{Bonnor's Stability Criterion}

For a polytropic, self-gravitating sphere of specific heat ratio $\gamma$, Bonnor's $(1956,1958)$ stability criterion 

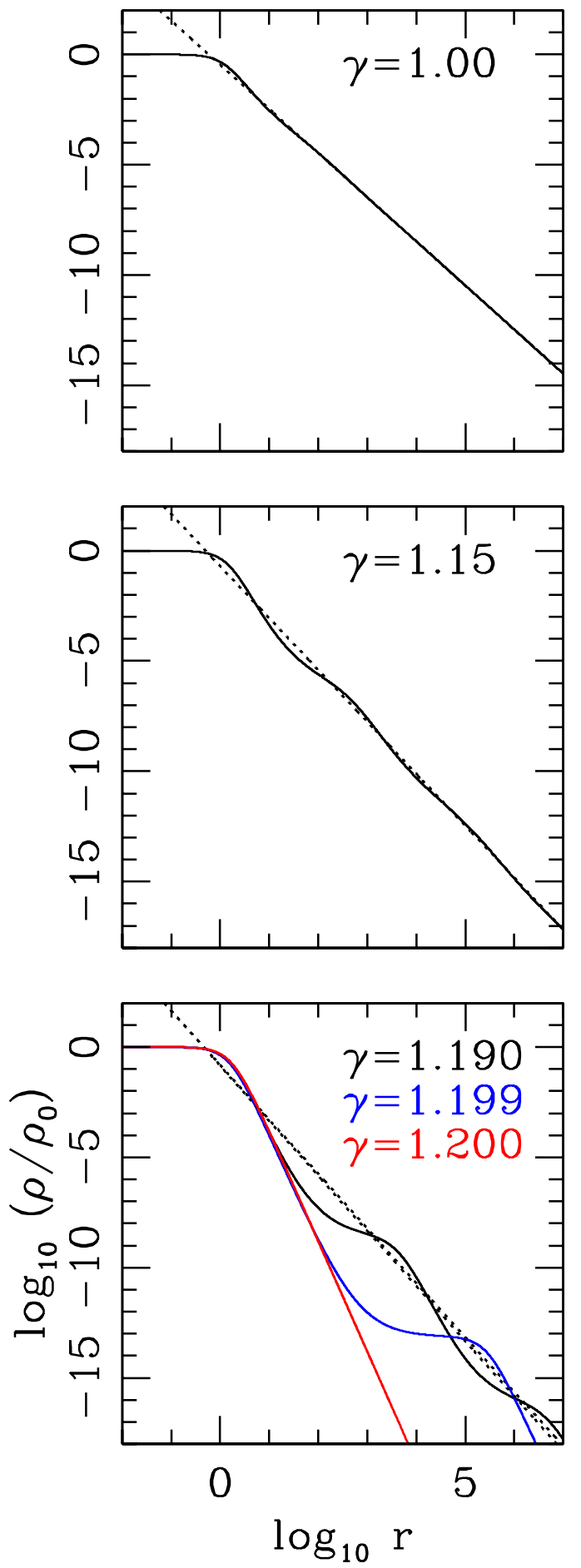

Fig. 1. The solid lines show the dimensionless nonsingular density vs. radius solutions for the isothermal, $\gamma=1$ case (top), for $\gamma=1.5$ center, and for $\gamma=1.190,1.199$ and 1.200 (bottom). The dashed curves show the $\gamma=1$, 1.15 and 1.2 singular solutions (in the top, center and bottom frames, respectively). The color figure can be viewed online.

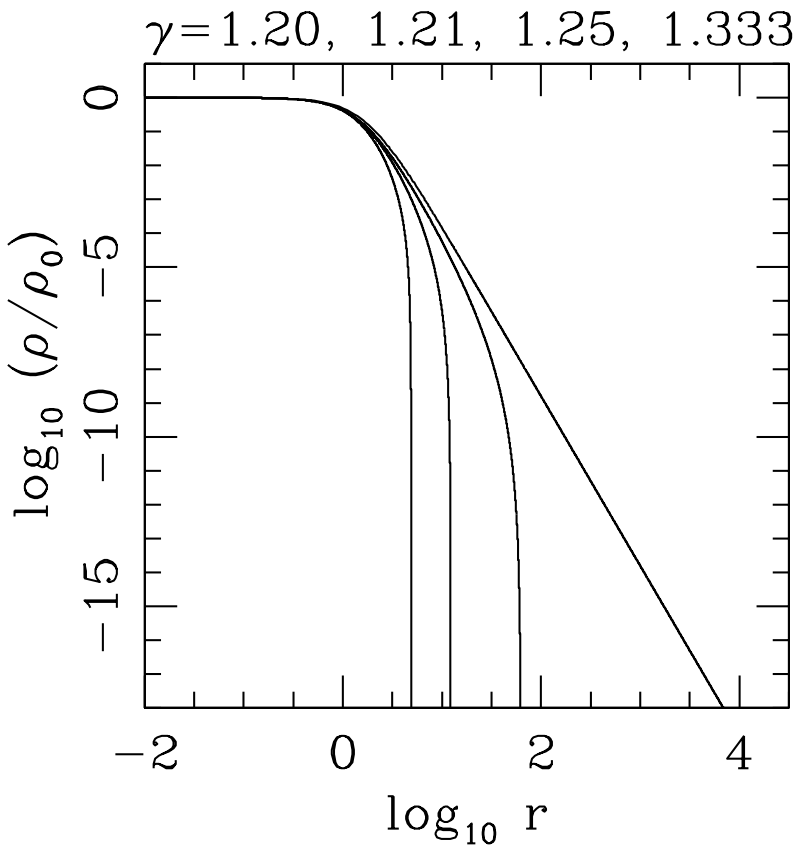

Fig. 2. Dimensionless nonsingular density $\left(\rho / \rho_{0}\right)$ vs. radius $\left(r=R / R_{c}\right.$, where $R_{c}$ is the core radius defined in equation 6 ) solutions for $\gamma=1.20,1.21,1.25$ and $1 . \hat{3}$. While the $\gamma=1.20$ solution goes out to infinite $r$, the solutions for larger $\gamma$ values reach zero densities at at an outer radius $r_{f}$ (which decreases for increasing $\gamma$ ).

can be written as:

$$
\begin{gathered}
\left(\frac{2 \pi G}{3 \gamma C}\right)^{1 /(2-\gamma)}\left(\frac{d \rho_{e}}{d R_{e}}\right)_{M=\text { const. }}= \\
\frac{r_{e} \dot{f}\left(r_{e}\right)}{1-\left(\frac{2-\gamma}{4-3 \gamma}\right) r_{e}^{\left(\frac{4-3 \gamma}{2-\gamma}\right)} \frac{f\left(r_{e}\right)}{F\left(r_{e}\right)}}-\frac{2 f\left(r_{e}\right)}{2-\gamma}<0,
\end{gathered}
$$

where $r_{e}=R_{e} / R_{c}$ is the dimensionless outer radius of the sphere,

$$
f(r)=r^{2 /(2-\gamma)} \rho^{\prime}(r) ; \dot{f}(r)=\frac{d f}{d r},
$$

and

$$
F(r)=\int_{0}^{r}\left(r^{\prime}\right)^{2(1-\gamma) /(2-\gamma)} f\left(r^{\prime}\right) d r^{\prime} .
$$

The derivation of equation (22) is completely analogous to the derivation of equation (17) of Raga et al. (2013) for the $\gamma=1$ case.

We then take the numerically derived $\rho^{\prime}(r)$ density stratifications described in $\S 3$, calculate $f(r)$, $\dot{f}(r)$ and $F(r)$ (equations 23-24), and then evaluate the term on the left-hand-side of the inequality of equation (22) to find the smallest radius $r_{s}$ at which 

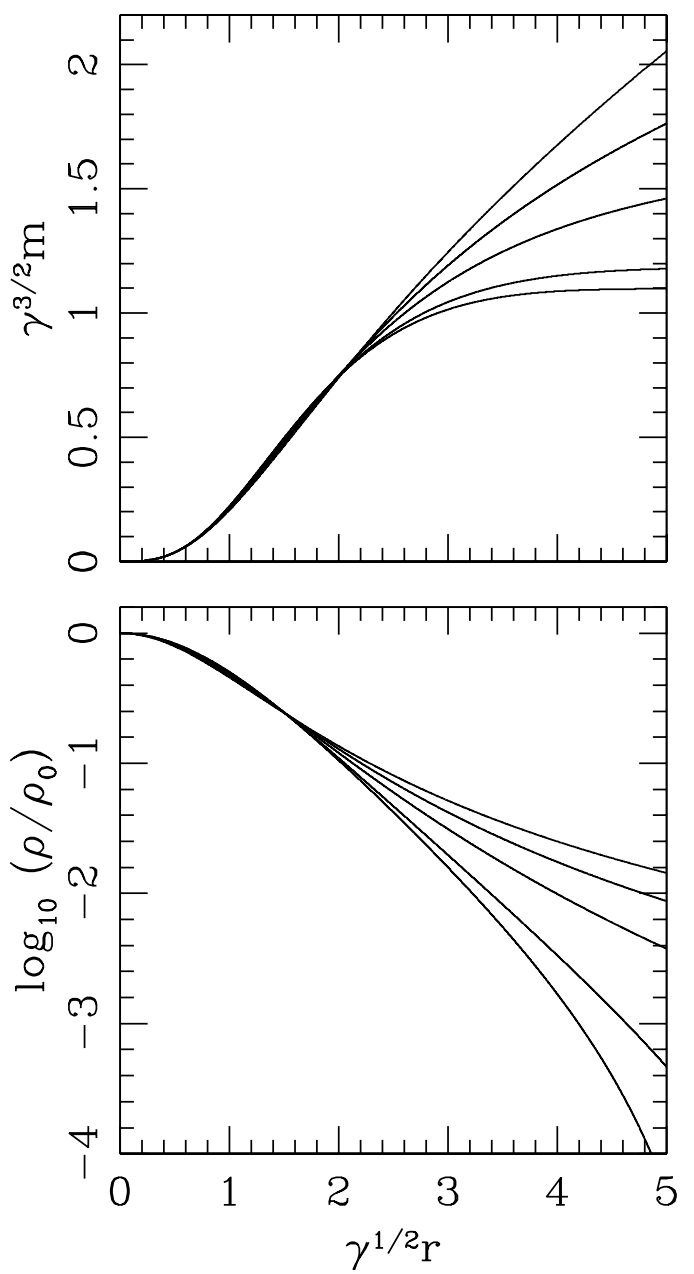

Fig. 3. Bottom frame: inner region of the nonsingular density vs. radius solutions for $\gamma=1.0,1.1,1.2$. 1.3 and 1.3. The solutions for higher $\gamma$ values have steeper density decreases at larger radii. Top frame: dimensionless mass $\gamma^{3 / 2} m=M /\left(\rho_{0} R_{c, 1}^{3}\right)$, where $\rho_{0}$ is the central density) as a function of radius. The mass distribution has lower growths at large radii for higher $\gamma$ values. Both the mass and the density profiles are shown as a function of $\gamma^{1 / 2} r=R / R_{c, 1}$ (where $R_{c, 1}$ is the core radius of the $\gamma=1$ solution with the same central sound speed, see the text).

the inequality is violated. In this way we derive the radius $r_{s}=R_{s} / R_{c}$ of the maximal stable sphere for a given value of $\gamma$.

\subsection{The Maximal Stable Solutions}

After determining the maximum radius for stability $r_{s}=R_{s} / R_{c}$ from Bonnor's criterion (equations 22$24)$, we calculate the dimensionless mass $m_{s}=m\left(r_{s}\right)$ and center-to-edge density ratio $\rho_{0} / \rho_{s}=1 / \rho^{\prime}\left(r_{s}\right)$ of the maximal stable solution. This exercise is re-
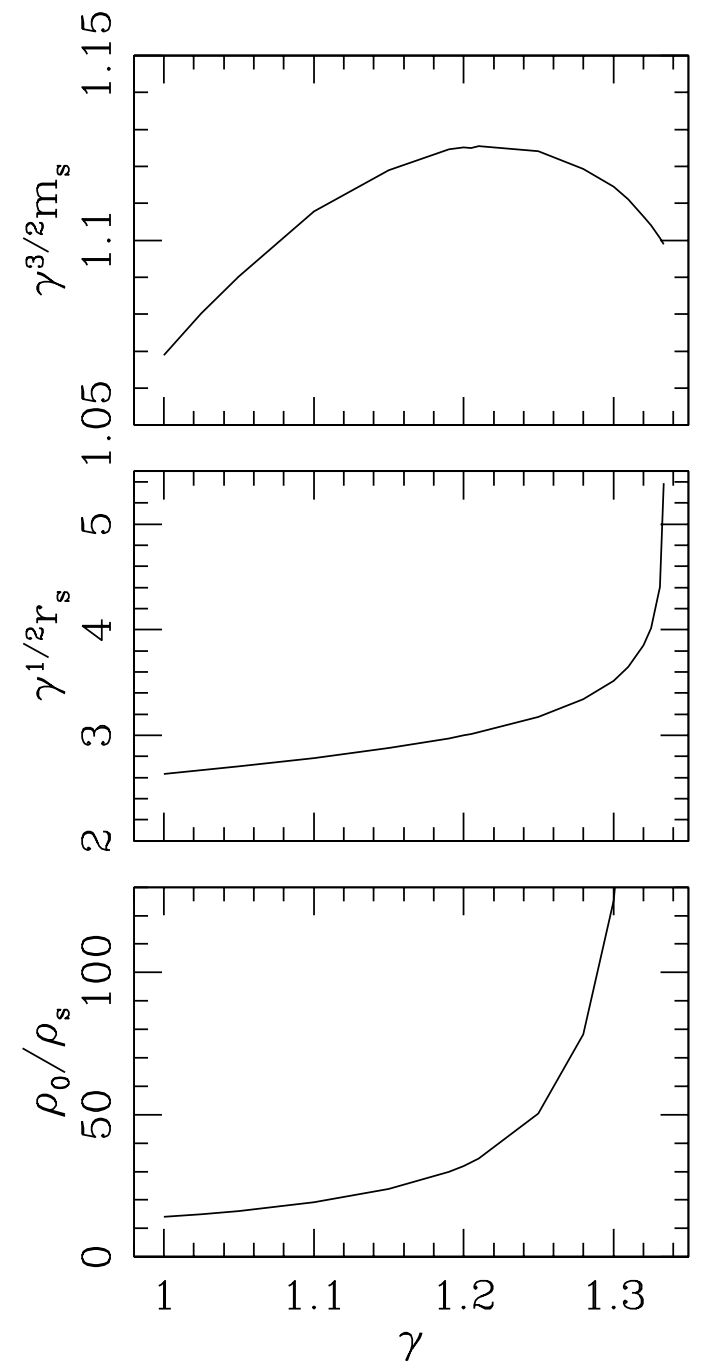

Fig. 4. Properties of the maximal stable solution as a function of $\gamma$. Bottom frame: center to edge density ratio $\rho_{0} / \rho_{s}$. Center: dimensionless outer radius $\gamma^{1 / 2} r_{s}=R_{s} / R_{c, 1}$. Top: dimensionless mass $\gamma^{3 / 2} m_{s}$ (see equation 12).

peated for several values of the specific heat ratio in the $\gamma=1 \rightarrow 4 / 3$ range. For $\gamma>4 / 3$, Bonnor's stability criterion is satisfied for all radii of the density stratifications (see also Bonnor 1958).

In Figure 4, we show the dimensionless mass $\gamma^{3 / 2} m_{s}=3 M_{s} /\left(4 \pi \rho_{0} R_{c, 1}^{3}\right)$ (see equations 11-12), outer radius $\gamma^{1 / 2} r_{s}=R_{s} / R_{c, 1}$, and center to edge density ratio $\rho_{0} / \rho_{s}$ of the maximal stable solution as a function of $\gamma$. We see that:

- $\rho_{0} / \rho_{s}$ grows as a function of $\gamma$, and reaches high values for $\gamma \approx 1.3$, finally diverging at $\gamma=4 / 3$, 

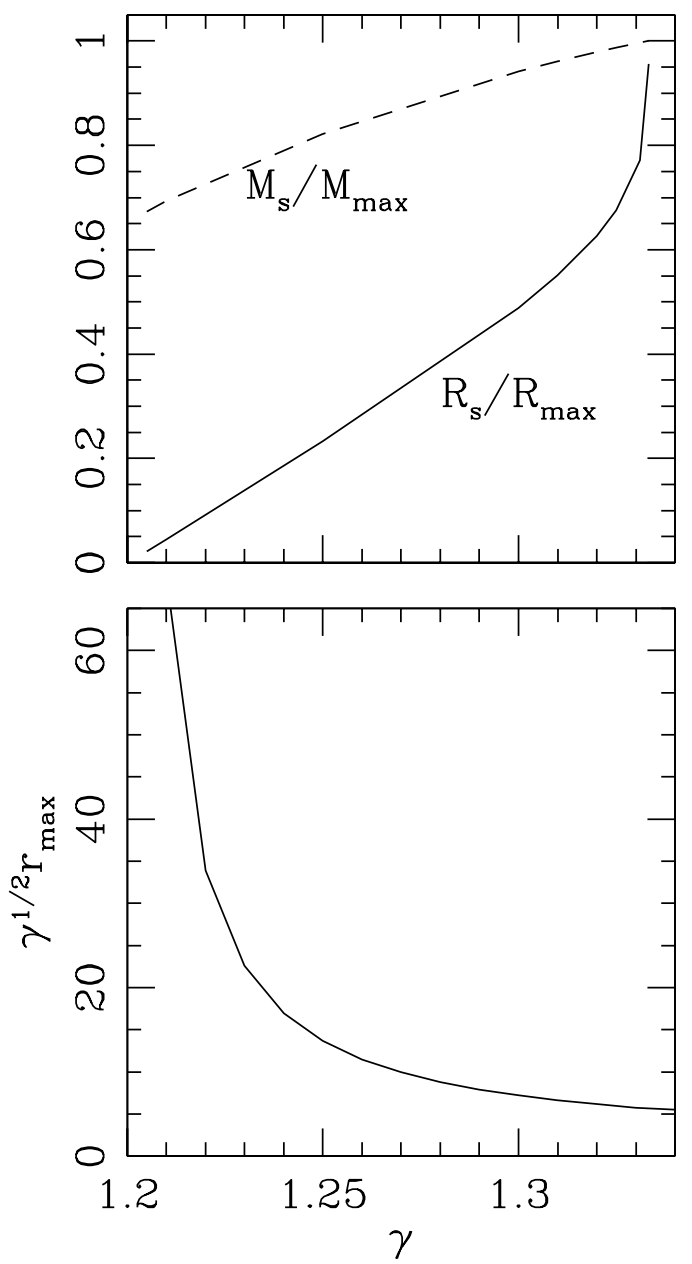

Fig. 5. Bottom frame: dimensionless maximum radius $\gamma^{1 / 2} r_{\max }=R_{\max } / R_{c, 1}$ as a function of $\gamma$. Top frame: ratios $M_{s} / M_{\max }$ and $R_{S} / R_{\max }$ of the mass $M_{s}$ and radius $R_{s}$ of the maximal stable solution to the maximum mass $M_{\max }$ and radius $R_{\max }$ of the polytropic spheres as a function of $\gamma$. We show the results obtained for $\gamma=(6 / 5)^{+} \rightarrow 4 / 3$, which have density distributions which go to zero at a maximum radius $R_{\max }$.

- $\gamma^{1 / 2} r_{s}$ monotonically grows as a function of $\gamma$, and reaches a $\gamma^{1 / 2} r_{s} \approx 5.4$ for $\gamma=4 / 3$,

- the dimensionless mass $\gamma^{3 / 2} m_{s}$ shows very small variations for $\gamma$ in the $1 \rightarrow 4 / 3$ range.

For the $6 / 5<\gamma \leq 4 / 3$ range (with density stratifications that end at an outer radius $R_{\max }$, see $\S 2$ and Figure 3), we obtain numerically the dimensionless outer radius $r_{\max }=R_{\max } / R_{c}$ and the mass $m_{\max }=m\left(r_{\max }\right)$ (see equation 12) of the full stratification. In Figure 5 , we plot the outer radius $\gamma^{1 / 2} r_{\max }=R_{\max } / R_{c, 1}$ and the ratios $M_{s} / M_{\max }$ and $R_{s} / R_{\text {max }}$ between the mass/radius of the maximal stable solution and the corresponding values of the full stratification.

From Figure 5 we see that:

- the outer radius $\gamma^{1 / 2} r_{\max }$ of the full stratification decreases monotonically from its infinite $\gamma=1.2$ value to a value of $\approx 5.63$ at $\gamma=4 / 3$,

- the $R_{s} / R_{\max }$ ratio increases from 0 (for $\gamma=1.2$ ) to 1 (for $\gamma=4 / 3$ ), with a faster increase as $\gamma=4 / 3$ is approached,

- the $M_{s} / M_{\max }$ ratio gradually increases from $\approx 0.65$ (for $\gamma=1.2$ ) to 1 (for $\gamma=4 / 3$ ).

Therefore, we see that the fraction of the total radius and mass of the finite, $\gamma>1.2$ self-gravitating spheres that is stable increases with $\gamma$. The (radial or mass) fraction that is stable tends to 1 as $\gamma=4 / 3$ is approached.

\section{SUMMARY}

We have studied the non-singular density stratifications that are solutions to the polytropic LaneEmden equation for self-gravitating spheres. We have first presented general analytical considerations about the proper adimensionalisation of the density and radius (using the second-order, small radius approximation to the non-singular density stratification), the derivation of the singular solutions, and the convergence at large radius of the nonsingular to the singular solutions (which occurs only for $\gamma<6 / 5)$. The results that have been presented (see $\S 2$ ) can be found in the previous literature (notably, in Chapter IV of the book of Chandrasekhar 1967 and in the book of Horedt 2004).

We have then computed numerical integrations of the Lane-Emden equation to obtain non-singular density stratifications for $\gamma$ in the $1 \rightarrow 4 / 3$ range (see $\S 3$ ). Solutions around $\gamma=1.2$ show the curious transition between the $\gamma<1.2$ density stratifications (which at large radii converge to the singular solutions) and the $\gamma=1.2$ stratification (which is analytic, and differs quite dramatically from the singular solution at large radii). It is not clear whether or not this rather curious mathematical feature has any relevance for astronomical applications.

Finally, we have applied Bonnor's stability criterion (for self-gravitating solutions of arbitrary $\gamma$, see equation 22) to our numerically obtained density stratifications. We find that for spheres with $\gamma=1 \rightarrow 4 / 3$ but with the same central temperature (or isothermal sound speed), the maximal stable sphere has (see Figure 4): 
- a center to edge density ratio that grows quite substantially with increasing $\gamma$, diverging for $\gamma \rightarrow 4 / 3$

- an outer radius that slowly grows with $\gamma$ in the $1 \rightarrow 1.2$ range, and has a steeper growth for $\gamma \rightarrow 4 / 3$,

- an almost constant mass as a function of $\gamma$.

Also, for the finite, $\gamma>6 / 5$ spheres, the maximal stable sphere has a mass and radius that grow with $\gamma$. For $\gamma>4 / 3$, the whole finite sphere is stable.

Applying these results to molecular clouds cores, we see that relatively small deviations from isothermality (which could be modelled with $\gamma$ values somewhat larger than 1) are not likely to lead to the formation of substantially different self-gravitating structures, in the sense that the maximal stable solutions will have almost the same mass (regardless of the value of $\gamma$ in the $1 \rightarrow 4 / 3$ range). The outer radius of the maximal stable sphere grows for $\gamma>1$, reaching a factor 1.5 larger value for $\gamma=1.2$. The largest effect is found for the center to edge density ratio, which grows by a factor of $\approx 2$ when $\gamma$ is changed from 1 to 1.2 and by a factor of $\approx 10$ when $\gamma=1.3$.

Therefore, we conclude that small deviations from isothermality will result in the production of maximal stable spheres with approximately the same mass, with somewhat larger outer radii, and with substantially higher center to edge density ratios. Clearly, it will be interesting to see whether the hydrodynamical collapse of the $\gamma=1 \rightarrow 4 / 3$ density stratifications resembles the well studied collapse of the isothermal $(\gamma=1)$ sphere.

We end by discussing the work of Kandori et al. (2005), who use near-infrared images to derive the column density distribution for a set of $\approx 10 \mathrm{Bok}$ globules. They carry out fits of the non-singular solution of the isothermal Lane-Emden equation to the derived structures. From these fits, they find the center to edge density ratios and the dimensionless outer radius $\xi_{\max }$ (which, due to a different adimensionalisation is a factor of $\sqrt{6}$ larger than the $\gamma^{1 / 2} r_{s}$ outer radius shown in our Figure 4) of the observed structures. Most interestingly, they find that while some of the globules of their sample are close to the stability limit, about half of them have center to edge density ratios and outer radii larger than the stability limit of the isothermal sphere. This result leads them to conclude that these globules above the isothermal stability limit are probably already collapsing.

Our models provide a possible alternative explanation for these results. As one can see from Figure 4, self-gravitating spheres with departures from isothermality (modeled as spheres with $\gamma>1$ ) have maximal stable solutions with center to edge density ratios and dimensionless outer radii larger than the ones of the maximal isothermal $(\gamma=1)$ solution. The higher center to edge density contrast globules observed by Kandori et al. (2005) could therefore correspond to stable self-gravitating spheres with such departures from isothermality.

AR acknowledges support from the DGAPAUNAM grant IG100218. JCRR acknowledges the Brazilian agency FAPESP grant 2017/12188-5. We thank an anonymous referee for pointing out the work of Kandori et al. (2005), which provides a most interesting connection between our models and reality.

\section{REFERENCES}

Bonnor, W. B. 1956, MNRAS, 116, 351 . 1958, MNRAS, 118, 523

Chandrasekhar, S. 1967, An introduction to the study of stellar structure (Dover, New York)

Falgarone, E., Puget, J. L. 1985, A\&A, 142, 157

Horedt, G. P. 2004, Polytropes - applicaations in astrophysics and related fields (Kluwer: Dordrecht) . 2013, ApJ, 773, 131

Hunter, C. 2011, MNRAS, 328, 839

Ito, Y., Poje, A., Lancelotti, C. 2018, NewA, 58, 15

Kandori, R., Nakajima, Y., Tamura, M. et al. 2005, AJ, 130,2166

Raga, A. C., Rodríguez-Ramírez, J. C., RodríguezGonzález, A., Lora, V., \& Esquivel, A. 2013, MNRAS, 49, 127

Shu, F. H. 1977, ApJ, 214, 488

R. S. Chan, A. Esquivel, J. A. Osorio-Caballero, A. C. Raga, and A. Rodríguez-González: Instituto de Ciencias Nucleares, Universidad Nacional Autónoma de México, Ap. 70-543, 04510 Cd. Mx., México (raga@nucleares.unam.mx)

V. Lora: Instituto de Radioastronomía y Astrofísica Teórica, Universidad Nacional Autónoma de México, Ap.3-72, 58089 Morelia, Michoacán, México.

J. C. Rodríguez Ramírez: Instituto de Astronomia, Geofísica e Ciências Atmosféricas, Universidade de São Paulo, R. do Matão 1226, 05508-090 São Paulo, SP, Brasil. 